\title{
4 PERCEPÇÃO dE PESSOAS QUE USAM DROGAS ACERCA DO TRATAMENTO EM UM CENTRO DE ATENÇÃO PSICOSSOCIAL
}

\author{
| Vanessa Manente ${ }^{1}$; Daiana de Siqueira ${ }^{2}$; Keity Soccol'; Silvana Andres; Janaina Canabarro ${ }^{5}$; Claudete Moreschi ${ }^{6}$ |
}

\section{RESUMO}

CONTEXTO: No Brasil, o Centro de Atenção Psicossocial representa um dos locais que é ofertado o tratamento às pessoas que usam drogas. OBJETIVO: Compreender como as pessoas que usam de drogas percebem o tratamento ofertado em um Centro de Atenção Psicossocial.

MÉTODO: Trata-se de uma pesquisa qualitativa, exploratória e descritiva, realizada com 21 usuários de álcool e drogas em tratamento no Centro de Atenção Psicossocial. A coleta de dados ocorreu no período de setembro e outubro de 2017, por meio de entrevista aberta.

RESULTADOS: Identificaram-se três categorias temáticas: tratamento pautado na abstinência; tratamento baseado na redução de danos e, estratégias para adesão ao tratamento. $\mathrm{O}$ tratamento mais referido pelos participantes foi a abstinência; a maioria dos participantes não acredita na efetividade da redução de danos; e, o medo da perda do vínculo com os familiares foi evidenciado como fator principal para adesão ao tratamento.

CONCLUSÃO: Notou-se a necessidade de maior participação dos usuários na escolha do seu tratamento, além da desmistificação acerca da abstinência e da redução de danos como opções de abordagens que contribuem com o tratamento das pessoas que são atendidas em um Centro de Atenção Psicossocial.

\section{PALAVRAS-CHAVE: Drogas; Redução do dano; Enfermagem; Transtornos relacionados ao uso de substâncias; Abstinência}

\section{RESUMEN}

\section{"Percepción de personas que usan drogas acerca del tratamiento} en un Centro de Atención Psicosocial“

CONTEXTO: en Brasil, el Centro de Atención Psicosocial representa uno de los locales que se ofrece el tratamiento a las personas que usan drogas. OBJETIVO: comprender cómo las personas que usan drogas perciben el tratamiento ofrecido en un Centro de Atención Psicosocial.

METODOLOGÍA: se trata de una investigación cualitativa, exploratoria y descriptiva, realizada con 21 usuarios de alcohol y drogas en tratamiento en el Centro de Atención Psicosocial. La recolección de datos ocurrió en el período de septiembre y octubre de 2017, por medio de una entrevista abierta.

RESULTADOS: Se identificaron tres categorías: tratamiento pautado en la abstinencia; tratamiento basado en la reducción de daños y estrategias para la adhesión al tratamiento. El tratamiento más mencionado por los participantes fue la abstinencia; la mayoría de los participantes no cree en la efectividad de la reducción de daños; y el miedo a la pérdida del vínculo con los familiares fue evidenciado como factor principal para la adhesión al tratamiento.

CONCLUSIÓN: Se notó la necesidad de mayor participación de los usuarios en la elección de su tratamiento, además de la desmitificación acerca de la abstinencia y la reducción de daños como opciones de abordajes que contribuyen con el tratamiento de las personas que son atendidas en un Centro de Atención Psicosocial.

DESCRIPTORES: Drogas; Reducción del daño; Enfermería; Trastornos relacionados con sustancias; La abstinencia

\begin{abstract}
"Perception of people who use drugs about treatment in a Psychosocial Care Center"

BACKGROUND: in Brazil, the Psychosocial Care Center represents one of the places that treatment is offered to people who use drugs.

AIM: to understand how people who use drugs perceive the treatment offered in a Psychosocial Care Center.

METHOD: this is a qualitative, exploratory and descriptive research carried out with 21 alcohol and drug users under treatment in the Psychosocial Care Center. The data collection took place in the period of September and October of 2017, through an open interview.

RESULTS: three categories were identified: treatment based on abstinence; treatment based on harm reduction, and strategies for adherence to treatment. The most mentioned treatment was abstinence; most participants do not believe in the effectiveness of harm reduction; and, fear of losing the bond with relatives was evidenced as the main factor for adherence to treatment.

CONCLUSION: It was noted the need for greater participation of the users in the choice of their treatment, besides the demystification about abstinence and harm reduction as options of approaches that contribute to the treatment of the people who are cared for in a Psychosocial Care Center.
\end{abstract}

KEYWORDS: Drugs; Harm reduction; Nursing; Centro de Atenção Psicossocial; Substance-related disorders; Abstinence

Submetido em 31-03-2018

Aceite em 13-07-2018

1 Enfermeira, Avenida Batista Bonoto Sobrinho, 97700-000 Santiago/RS, Brasil, vanessabatistam@hotmail.com.

2 Enfermeira; Doutora em Enfermagem; Docente na Universidade Regional Integrada do Alto Uruguai e das Missões, Erechim - RS, Brasil, daianasiqueira@yahoo.com.br

3 Enfermeira; Doutora em Enfermagem; Docente no Centro Universitário Franciscano, 97010-030, Santa Maria - RS, Brasil, keitylais@hotmail.com

4 Enfermeira; Mestre em Enfermagem; Docente na Universidade Regional Integrada do Alto Uruguai e das Missões, Erechim - RS, Brasil, silvana.andres@yahoo.com.br

5 Enfermeira no Hospital Geral do Estado de Santa Maria-RS, 97010-290 Passo D’areia, Santa Maria - RS, Brasil, jana.l.c2010@hotmail.com

6 Enfermeira; Doutora em Ambiente e Desenvolvimento; Docente na Universidade Regional Integrada do Alto Uruguai e das Missões, Brasil, clua_moreschi@yahoo.com.br

Citação: Manente, V. B., Siqueira, D. F., Soccol, K. L. S., Andres, S. C., Canabarro, J. L., \& Moreschi, C. (2018). Percepção de pessoas que usam drogas acerca do tratamento em um Centro de Atenção Psicossocial. Revista Portuguesa de Enfermagem de Saúde Mental (20), 27-33. doi: 10.19131/rpesm.0223 


\section{INTRODUÇÃO}

No Brasil, dentre os serviços que prestam assistência à saúde das pessoas que usam drogas situam-se os Centros de Atenção Psicossocial (CAPS), que são regulamentados pela Portaria GM/MS no 336/2002. Esses serviços prestam assistência às pessoas com transtornos decorrentes do uso e dependência de drogas (Gabatz, Johann, Terra e Padoin, 2013).

Essa Portaria, por meio da inserção de novos serviços de saúde mental, garantiu uma mudança no cenário do atendimento a essas pessoas, que antes eram atendidos principalmente em hospitais psiquiátricos (Gabatz et al., 2013). Cada CAPS, estabelece o plano terapêutico de acordo com a individualidade de cada usuário levando em consideração a sua autonomia, podendo estar voltado para a prevenção à recaída ou para a redução de danos.

As estratégias de redução de danos, desde sua inserção no Brasil, provocaram polêmicas e resistências, sob a alegação de facilitar o uso/consumo de drogas e de levar a gastos indevidos do dinheiro público. A redução de danos pode ser definida como um modo diferente de abordar o tratamento do usuário de álcool e drogas, descentralizando o foco do problema da erradicação e da abstinência e privilegiando o direito à saúde de todos e o respeito à liberdade individual daquele que não deseja ou não consegue interromper o uso da substância (Machado e Boarini, 2013).

Já a abstinência, ou abstenção do uso de drogas, ocorre quando o usuário por razões pessoais cessa o uso da substância que vinha consumindo. A pessoa que pratica a abstinência de álcool é chamada de abstêmio. Considera-se em abstinência aquela pessoa que não ingeriu bebidas alcoólicas nos últimos doze meses. O termo abstinência não deve ser confundido com síndrome de abstinência. É possível que qualquer usuário problemático de drogas permaneça em abstinência, porém isso vai depender do seu estado psíquico e das circunstâncias em que está inserido (Secretaria Nacional de Políticas sobre Drogas, 2010).

As ações de redução de danos levam em consideração o respeito aos direitos humanos e tem suas ações voltadas em prol da população de usuários de drogas e seus familiares (Elias e Bastos, 2011). A redução de danos caracteriza-se como uma medida que não estabelece como meta inicial para o tratamento a abstinência do uso drogas, mas pressupõe que a atenção à saúde chegue até o usuário, onde quer que ele se encontre, e não o contrário (Brasil, 2014).
Enquanto a abstinência apresenta uma proposta de remissão do sintoma e a cura do doente, a redução de danos está direcionada para a produção de saúde, incentivando a autonomia do cuidado de si (Passos e Souza, 2011). O Brasil, ainda hoje, prevê essas duas modalidades no tratamento dos usuários de álcool e outras drogas, que envolvem a abstinência e a redução de danos.

Diante do exposto, observou-se que a escolha entre a redução de danos e a abstinência, é algo pactuado com a própria pessoa e que essa opção é pessoal e individual, respeitando a sua autonomia e direito de escolha. Assim, o presente estudo apresenta como objetivo compreender como as pessoas que usam de drogas percebem o tratamento ofertado em um Centro de Atenção Psicossocial.

Considerando o contexto acima, questionou-se: como as pessoas que usam drogas percebem o tratamento ofertado em um Centro de Atenção Psicossocial?

\section{MÉTODOS}

Trata-se de uma pesquisa de abordagem qualitativa, exploratória e descritiva. A pesquisa qualitativa está preocupada com a realidade, com os significados, com os motivos, crenças, valores e atitudes que as pessoas atribuem a um determinado fenômeno. Esse tipo de pesquisa pode ser aplicada tanto para compreender as relações, as percepções, opiniões e interpretações que os sujeitos fazem a respeito de como vivem, constroem relações e a si mesmos, como sentem e pensam sob a ótica do próprio sujeito (Minayo, 2014).

A presente pesquisa foi realizada em um Centro de Atenção Psicossocial localizado em um município do Estado do Rio Grande do Sul - Brasil. A escolha desse cenário ocorreu em virtude de que esse CAPS além de atender pessoas com transtornos mentais, atende também pessoas com transtornos relacionados ao uso de álcool e outras drogas.

O CAPS possui uma equipe composta por uma enfermeira, dois técnicos de enfermagem, quatro psicólogas, uma médica psiquiatra, além dos oficineiros. Desenvolvem atividades de educação em saúde com as pessoas que usam drogas licitas e ilícitas e com as pessoas que apresentam transtornos mentais.

Os participantes da pesquisa foram 21 usuários que estavam na modalidade de tratamento não intensivo que faziam uso de drogas lícitas e/ou ilícitas, em diferentes estágios e tempo da evolução do tratamento, no referido serviço durante o período da coleta dos dados. 
Como critérios de inclusão, elencou-se aqueles maiores de 18 anos que estavam inseridos em alguma modalidade de tratamento no referido serviço. E, os critérios de exclusão foram os indivíduos que possuíam dificuldades de comunicação que viesse a prejudicar a condução da entrevista.

Para a produção dos dados foi utilizado a entrevista aberta, por meio da qual os participantes tiveram a possibilidade de discorrer sobre o tema proposto. Para que as entrevistas deste estudo alcançassem os objetivos propostos, foi utilizado um instrumento para enunciação dos dados de identificação dos participantes da pesquisa, e as seguintes questões iniciais abertas: Como você percebe o tratamento voltado para a abstinência? O que você entende por redução de danos? E o que você pensa sobre a redução de danos? Quais as estratégias que você percebe na adesão do seu tratamento? Salienta-se que todos os participantes realizavam tratamento recorrente no serviço, ou seja, nenhum participante estava realizando o seu primeiro tratamento.

As entrevistas aconteceram no período entre os meses de setembro a outubro de 2017 , sendo realizadas individualmente, em uma sala previamente reservada no CAPS, com antecedente agendamento da entrevista em horário definido em acordo com sua disponibilidade. As informações emergidas durante as entrevistas foram registradas em um gravador digital, conforme autorização prévia do participante

Para a análise dos dados, foi utilizada a Análise Temática seguindo a Proposta Operativa de Minayo que se caracteriza por dois níveis de interpretação. O primeiro momento interpretativo é constituído pelo mapeamento do campo das determinações fundamentais na fase exploratória, que se refere ao contexto histórico do grupo social em questão. O segundo momento interpretativo consiste no ponto de partida e de chegada da pesquisa, representa a convergência com os fatos empíricos, é o momento em que se encontra nos relatos dos informantes o sentido, a lógica interna, as projeções e as interpretações, havendo um significado cultural do grupo e um elo muito mais abrangente. Para operacionalizar esse segundo momento é sugerido os seguintes passos: pré-análise, exploração de material e tratamento dos resultados obtidos e interpretações (Minayo, 2014). Para a execução e desenvolvimento desta pesquisa foram respeitados os preceitos éticos da Resolução No 466/12 do Conselho Nacional de Saúde (Brasil, 2012). Recebeu aprovação do comitê de ética sob número do parecer 2.222.950 e CAEE 72771417.6.0000.5353.
Para preservar a identidade dos sujeitos foi utilizada a letra ' $U$ ', que é a letra inicial da palavra participante seguida de um numeral (U1, U2, U3, e assim sucessivamente).

\section{RESULTADOS}

Participaram do estudo 21 usuários, sendo que 02 eram do sexo feminino e 19 do sexo masculino. Destes, 14 participantes com idades entre 40 e 60 anos, 05 com idades entre 20 e 40 anos e 02 com idade acima de 60 anos. Em relação a escolaridade, 16 participantes com ensino fundamental incompleto, 01 com ensino fundamental completo, 03 com ensino médio completo e 01 analfabetos. Todos os entrevistados realizavam tratamento no serviço em regime não intensivo e participavam do "Grupo Álcool e drogas", que acontecia nas quintas-feiras, no turno da manhã.

A análise das falas das pessoas que usam drogas possibilitou a compreensão das seguintes categorias temáticas: Tratamento pautado na abstinência; Tratamento baseado na redução de danos; e, Estratégias para adesão ao tratamento.

\section{Tratamento Pautado na Abstinência}

O tratamento mais referido pelos participantes foi a abstinência. Alguns participantes relataram que percebem necessidade em cessar com o uso da substância, pois assim se sentem melhor e com mais autocontrole.

Estou indo até o último e não bebo. Não quero beber mais. O tratamento eu vou fazendo e não tem bebida para mim. (E1)

Parar é um tempo, e eu decidi deixar. Estou me sentindo muito bem. Melhora tudo na vida da gente. Em casa, com os familiares. Com as pessoas, todo mundo respeita. (E3)

Deve fazer uns cinco ou seis meses que não bebo. Não bebi mais. Nem cerveja. Só que eu não sabia beber uma ou duas e parar, não me controlava. (E17)

Ainda, relataram que embora pratiquem a abstinência, ainda em determinados períodos, sentem vontade de ingerir algum tipo de substância.

Depois que eu parei de tomar, nunca mais tentei colocar na boca. Às vezes, até dá uma vontade, mas olho e penso que se eu tomar, volta o vício. Aí não, deixo quieto. (E13)

Eu cheguei num bar comprar cigarro, aí me deu vontade de comprar um samba, mas aí um amigo me disse que não era para beber. Aí peguei o cigarro e fui embora. Não comprei. (E14) 


\section{Tratamento Baseado na Redução de Danos}

A redução de danos foi vista como uma forma de tratamento com bons resultados por alguns participantes. Alguns relataram estarem praticando ou já terem praticado redução de danos, mesmo que de forma desconhecida.

Eu acho que dá certo. Não é o meu caso, porque não tenho vontade nenhuma. Mas se eu ainda bebesse, acho que seria bom. (E15)

Estive dois anos e meio sem colocar nada na boca, aí tive uma pequena recaída, e às vezes, eu coloco uma cerveja na boca. Mas cerveja. Em algum final de semana só. (E5)

Usei e uso ainda. Estou usando. Estou usando maconha. Porque eu não consigo ficar sem. (E9)

Uso ali, um ou dois dias e paro dois ou três meses. Um ano já fiquei sem. Uso maconha, cocaína e crack. Nessas duas semanas que comecei a vir aqui, não usei. (E18) Porém, a maioria dos participantes não acredita na efetividade da redução de danos, usando como uma das formas de justificativa o descontrole no consumo.

Não tinha como dizer assim: vou tomar hoje e amanhã não vou tomar. No outro dia, amanhecia com vontade de tomar mais ainda. Não tinha como diminuir. (E2) Não dá para só diminuir. Tu diminui e quando vê está do mesmo jeito. Eu acho que não. (E6)

Tem que parar de beber completamente. Para mim não funciona. Dizer que diminui é bobagem. (E7)

Não existe isso, para mim não existe. É para pouquíssimos que existe. Dizer que hoje vai tomar só um traguinho. Porque quem é viciado, eu tiro por mim, não pode tomar um gole. Se tomar o primeiro, não para mais. (E10)

Para mim não dá certo. Se eu fizer isso, inventar de seguir tomando, mesmo que seja pouquinho, eu não aguento. Aí vou virar na mesma pessoa que eu era. (E16)

Redução eu acho que não. Tem gente que não se controla. Eu mesmo sou um. Se eu começar a tomar, tenho que tomar muito. (E1)

\section{Estratégias para Adesão ao Tratamento}

A família foi vista pelos usuários como um ponto positivo, representando uma estratégia para adesão ao tratamento. O medo da perda do vínculo e o distanciamento dos familiares também foi evidenciado como fator motivador para adesão ao tratamento.
No meu ponto de vista, a pessoa tem que colocar um capricho, ela mesma tem que pensar nela. Eu penso em mim, penso na minha filha. Eu penso na minha vida e na minha filha. Por isso eu deixei de beber. (E1)

Olha, agora eu penso no serviço. Em me firmar no serviço. E na minha família. A minha família é muito importante também. (E10)

Decidi parar porque tinha que cuidar da minha mãe, ela morava sozinha. Daí decidi não beber mais. (E16)

Eu tenho esposa, filhos. O medo de perder a família, o ambiente familiar. Ter que ficar longe, afastado. Porque a minha esposa não me aceita drogado em casa. Se ela vê que eu estou drogado, já manda eu sair. (E18)

Outros participantes identificaram como estratégias para a adesão ao tratamento o uso de medicamentos para ansiedade e as internações em unidades psiquiátricas e fazendas terapêuticas.

Eu fui para a fazenda. Eu tive que ir. Foi muito bom para mim. (E4)

Tomo medicamento para me acalmar, para ficar bem. Por exemplo, com esse medicamento e os tratamentos que eu fiz, que eu estou fazendo, não tenho vontade. (E4)

Eu disse que não tinha mais para onde ir, que trabalhar eu não podia. Tive um câncer na boca. Fiquei pensando o que iria fazer da vida. Chamei a minha cunhada e pedi para ela me internar de novo. (E6)

Através da medicação, para a gente não achar falta. $\mathrm{E}$ agora, a psicóloga arrumou para eu ir para a fazenda terapêutica, ficar um ano, para largar de vez. (E8)

Salienta-se que os participantes relataram outras estratégias para adesão, como o consumo de algumas bebidas e de cigarro como forma de suprir a necessidade do uso das substâncias psicoativas. Com relação ao consumo de outras bebidas, as mais consumidas segundo os relatos foram o chimarrão e o refrigerante.

Levanto cedo e tomo bastante mate. Estando em casa, fico quase todo o tempo com a cuia na mão. (E2)

Quando eu sinto vontade de beber, tomo refrigerante, água. Faço outras coisas para passar o tempo. (E11)

Foi aos poucos para deixar. Foi bem complicado. Nem mesmo aquelas sem álcool eu tomo. Eu tomo coca cola, guaraná, tomo suco também. Mas bebida não. (E12)

Chego nos bares e compro uma coca pequena, só para matar o tempo. Não preciso mais ficar bebendo. Outra coisa que eu faço é fumar. (E19) 


\section{DISCUSSÃO}

Constatou-se que a forma de tratamento que os participantes mais buscam no Centro de Atenção Psicossocial é a abstinência. $\mathrm{O}$ tratamento pautado na abstinência, ou seja, na proibição total do consumo de substâncias psicoativas constitui apenas uma entre as muitas formas de lidar com o uso dessas substâncias e não tem se mostrado a mais eficiente (Trad, Trad e Romaní, 2013). Considerar a abstinência como o principal objetivo do cuidar ao usuário de substâncias psicoativas pode limitar as opções de trabalho dos profissionais de saúde, visto que em muitos casos os usuários não se reconhecem como tal, o que dificulta o sucesso do tratamento (Dourado et al., 2014).

Evidenciou-se que os usuários sentem necessidade em parar com o uso da substância, pois assim se sentem melhor e com mais autocontrole. A decisão de consumir ou não é mediada pelas expectativas que a pessoa tem quanto aos efeitos iniciais do consumo da substância. Se inicialmente a ênfase é dada à manutenção da abstinência, posteriormente devem ser abordados aspectos disfuncionais da personalidade, visando uma maior adesão ao tratamento e uma melhor capacitação para a resolução de problemas (Silva, 2015).

Ainda, nesse sentido, na abstinência, os usuários em determinados períodos, sentem vontade de ingerir algum tipo de substância. A possibilidade de surgir uma recaída pode ocorrer quando as estratégias usadas pela pessoa para manter o estado de abstinência falham e, consequentemente, venham a apresentar sentimentos de fracasso, desesperança e culpa. É possível que possa afetar negativamente a sua autoeficácia (Silva, 2015).

Para a adesão ao tratamento, a importância da família e o medo do distanciamento dos familiares foi evidenciado. A dependência química de um ou mais membros da família causa um impacto importante nas relações afetivas interpessoais e sociais. Assim, dificulta a aproximação e manutenção de laços de intimidade, de confiança, com reflexos na interação do familiar com a sua rede social e com o usuário (Maciel, Zerbetto, Filizola, Dupas e Ferreira, 2013).

O isolamento familiar pode levar ao comprometimento do tratamento, além de dificultar a ação do profissional envolvido no processo de prevenção e promoção da saúde mental. Dessa maneira, a interação da família no tratamento do usuário é um fator que merece investimento, seja em relação ao atendimento individualizado, ou por meio de intervenções no âmbito comunitário para a integração social da família.
O envolvimento de famílias que compartilham do mesmo problema torna-se de grande relevância para a terapêutica, por criar um espaço de trocas de vivências, angústias e informações para a compreensão da dependência química (Maciel et al., 2013).

Com relação aos modelos de tratamento, após a criação da Política de Atenção Integral ao Usuário de Álcool e Outras Drogas, em 2003 e, na Política Nacional sobre Drogas, em 2005, houve o surgimento da redução de danos (Brasil, 2003). O tratamento com base na redução de danos tem como pilar a liberdade de escolha do indivíduo, visando à sua responsabilidade e colaboração no gerenciamento do próprio consumo de forma menos prejudicial à saúde (Trad et al., 2013).

No decorrer das abordagens realizadas, os participantes mostraram desconhecer o tratamento voltado à redução de danos. Alguns participantes não acreditam na efetividade da redução de danos, usando como uma das formas de justificativa o descontrole no consumo. Esta percepção pode resultar do pavor que o tema provoca no imaginário popular. Assim, coloca em risco as importantes experiências inovadoras apresentadas como possibilidades de tratamento no Brasil, em um campo em que ainda existe vasto caminho a percorrer (Dourado et al., 2014).

Sabe-se que não é submetendo o ser humano à imposição de que seja "abstêmio por natureza" que se solucionará o problema. A abordagem de redução de danos está mais próxima da visão atual que a ciência tem acerca do uso de substâncias psicoativas do que a abordagem proibicionista. Considera-se que práticas sociais, dependem mais das estruturas e das configurações existentes na sociedade em que são empregadas do que apenas da ação farmacológica de uma substância (Trad et al., 2013).

Em contrapartida, a redução de danos foi visualizada por alguns participantes como uma forma de tratamento efetiva. Assim, a redução de danos se estabelece como ferramenta de tratamento capaz de mitigar as consequências maléficas para o organismo do usuário. Além disso, atua como estratégia, na medida em que a própria difusão da informação já é elemento responsável por minimizar os danos e consequências sociais para os usuários (Dourado et al., 2014).

É fundamental informar/orientar os usuários acerca das consequências negativas decorrentes do uso de substâncias psicoativas, bem como sobre a prevenção do abuso das mesmas, visto que essas podem provocar sequelas ao organismo. 
Ainda, é imprescindível informá-los a respeito das formas e estratégias de tratamento, assim como informar aos não usuários acerca das reais consequências das drogas, preparando-os para melhor lidar com a situação. Evita-se, assim, a continuação de um discurso perpetrado pelo medo, pelo proibicionismo, pelo abstencionismo e pela ignorância (Silva, 2015).

Nesse cenário, a abordagem de redução de danos propõem estratégias que se adequem aos seus interesses, protegendo a sua vida e abrandando seu sofrimento. Além disso, não é necessário considerar este método como único caminho a ser adotado, na medida em que, em sua essência, ele não estabelece qualquer contradição ao tratamento associado a outras estratégias. É possível traçar planos terapêuticos que compatibilizem a redução de danos com outros tratamentos, como, por exemplo, os grupos de ajuda mútua, não havendo qualquer prejuízo ou empecilho a essa (ou outra) associação (Silva, 2015). Além disso, é importante fortalecer a articulação da rede de atenção em saúde com o intuito de facilitar o acesso e tratamento nos serviços (Wandekoken e Siqueira, 2013).

Salienta-se que a redução de danos deve ser compreendida como uma estratégia política de saúde pública que se apresenta diante da impossibilidade de uma sociedade sem substâncias. Deste modo, apresenta-se como uma forma pragmática para lidar com aquelas pessoas que estão em situação de risco ou sofrimento em decorrência do seu uso (Dourado et al., 2014).

\section{CONSIDERAÇÕES FINAIS}

Ao compreender como os usuários de drogas percebem o tratamento ofertado em um Centro de Atenção Psicossocial, evidenciou que a abstinência é o modelo de tratamento mais utilizado pelos participantes. Alguns participantes acreditam na efetividade da redução de danos e praticam essa modalidade de tratamento mesmo que de forma desconhecida. Outros, porém, não acreditam que a redução de danos possa ser efetiva. A família foi citada pelos participantes como a principal estratégia para adesão ao tratamento. Notou-se a necessidade de maior participação das pessoas que usam drogas na escolha do seu tratamento, de modo que possam realizar suas escolhas, decidir sobre o modelo de tratamento que vão seguir, baseado em orientações realizadas pelos profissionais de saúde.

\section{IMPLICAÇÕES PARA A PRÁTICA CLÍNICA}

Espera-se que esse estudo venha contribuir com a desmistificação acerca dos dois tipos de abordagem que direcionam o tratamento dos usuários de drogas, bem como, possibilitar aos usuários e aos serviços de saúde desenvolver uma melhor reflexão acerca da temática.

\section{REFERÊNCIAS BIBLIOGRÁFICAS}

Brasil. (2003). A Política do Ministério da Saúde para atenção integral a usuários de álcool e outras drogas / Ministério da Saúde, Secretaria Executiva, Coordenação Nacional de DST e Aids. Brasília: Ministério da Saúde.

Brasil. (2014). O uso de substâncias psicoativas no Brasil: módulo1. (5 $5^{\text {a }}$ ed). Brasília: Secretaria Nacional de Políticas Públicas sobre Drogas.

Brasil. (2012). Resolução 466/12- Trata de pesquisas em seres humanos e atualiza a resolução 196. Disponível em: http://conselho.saude.gov.br/resolucoes/2012/ Reso466.pdf

Dourado, G.O.L., Costa, M.C.M., Soares, A.D.A.S., Queiroz, A.A.F.L.N., Sousa, A.F.L.S., e Carvalho, L.K.C.A. (2014). Redução de danos no âmbito da Estratégia Saúde da Família: análise reflexiva. Revista Interdisciplinar. 7 (2):173-183.

Elias, L.A., e Bastos, F.I. (2011). Saúde Pública, Redução de Danos e a Prevenção das Infecções de Transmissão Sexual e Sanguínea: revisão dos principais conceitos e sua implementação no Brasil. Ciência \& Saúde Coletiva, 16 (12):4721-4730. Doi: 10.1590/S141381232011001300021

Gabatz, R.I.B., Johann, M., Terra, M.C., e Padoin, S.M.M. (2013). Percepção do usuário sobre a droga em sua vida. Escola Anna Nery, 17 (3), 520-525. Doi: 10.1590/S1414-81452013000300016

Machado, L.V., e Boarini, M.L. (2013). Políticas Sobre Drogas no Brasil: a Estratégia de Redução de Danos. Psicologia: Ciência e Profissão, 33 (3), 580-595. Doi: $10.1590 /$ S1414-98932013000300006 
